\title{
El ladrillo en la arquitectura ecléctica y modernista de la ciudad de Zamora: análisis de los tipos, los aparejos y la ejecución de los muros
}

\section{Bricks in the eclectic modernist architecture of the City of Zamora (Spain): an analysis of the types, building tools used and the construction of the walls}

M. A. Rodríguez-Esteban ${ }^{(*)}$, M. S. Camino-Olea ${ }^{(* *)}, \underline{\text { M. P. Sáez-Pérez }}{ }^{(* *)}$

\section{RESUMEN}

El presente trabajo lleva a cabo una investigación sobre los tipos de ladrillos empleados en las fachadas de los inmuebles de la época decimonónica de la capital zamorana. Este estudio engloba no sólo los ladrillos sino la relación de los mismos con los usos de los inmuebles. El análisis desarrollado es fundamentalmente técnico, centrándose tanto en los aspectos materiales y morfológicos de las piezas como en la relación existente entre los mismos y los diferentes sistemas constructivos empleados en la ejecución de sus muros.

La existencia de múltiple y variada documentación original, combinada con la información recabada in situ sobre los inmuebles que se conservan en la ciudad, ha permitido realizar un estudio exhaustivo y detallado, que se destaca como el objetivo primordial, pues permitirá desarrollar de cara a las futuras actuaciones de conservación, intervenciones para preservar y actuar sobre los elementos de interés.

Palabras clave: Ladrillo; Zamora; S. XIX-XX; aparejos.

\section{ABSTRACT}

The present work studies the types of bricks used on the façades of the $19^{\text {th }}$ century buildings of the City of Zamora. The study covers not only the bricks themselves but also their relationship with the use the buildings were put to. The analysis is essentially technical, focusing on both the material and morphological aspects of the bricks and the relationship between them and the different construction systems used to build the walls.

The existence of abundant and varied original literature, together with information collected "in situ" about the buildings preserved in the City, has allowed an exhaustive study that stands out as an initial aim since it will offer a way to guide future actions of conservation and interventions aimed at preserving the elements of interest.

Keywords: Brick; Zamora; $19^{\text {th }}$ and $20^{\text {th }}$ centuries; bonding.

(*) Universidad de Salamanca (España).

(**) Universidad de Valladolid (España).

(***) Universidad de Granada (España).

Persona de contacto/Corresponding author: mpsaez@ugr.es (M. P. Sáez-Pérez)

Cómo citar este artículo/Citation: Rodríguez-Esteban, M.A., Camino-Olea, M.S., Sáez-Pérez, M.P. (2014). El ladrillo en la arquitectura ecléctica y modernista de la ciudad de Zamora: análisis de los tipos, los aparejos y la ejecución de los muros. Informes de la Construcción, 66(535): e035, doi: http://dx.doi.org/10.3989/ic.13.053.

Licencia / License: Salvo indicación contraria, todos los contenidos de la edición electrónica de Informes de la Construcción se distribuyen bajo una licencia de uso y distribución Creative Commons Reconocimiento no Comercial 3.o. España (cc-by-nc). 


\section{INTRODUCCIÓN}

La ciudad de Zamora forma parte de ese grupo de ciudades españolas que en las últimas décadas del siglo XIX y primeras del XX fueron objeto de una importante transformación arquitectónica.

Fue una etapa brillante, presidida por un nuevo estilo que en aquella época se desplegaba por todo el territorio nacional, el eclecticismo. Esta corriente estuvo marcada, entre otros aspectos, por la utilización de nuevos materiales de construcción entre los que destacan, de forma especial, los ladrillos, que con un novedoso sistema de fabricación industrial, fueron utilizados como principal recurso matérico en la ejecución de los muros de las fachadas.

Sin embargo, esta transformación adquiere mayor relevancia en este municipio castellano, por un lado porque se llevó a cabo una amplia revitalización arquitectónica sobre una ciudad que había vivido en una evidente atonía en las centurias anteriores. Por otro, porque se produjo un cambio sustancial en la manera de construir, ya que la piedra arenisca del país que se había utilizado como material principal en la ejecución de los muros, a finales del siglo XIX fue radicalmente desbancada por las piezas de arcilla cocida. A partir de esta centuria, el ladrillo que era completamente novedoso en esta ciudad comienza a aparecer en las construcciones, instaurándose como el material constructivo y casi ornamental por excelencia, siendo reiteradamente utilizado en la ejecución de las fachadas.

Por lo tanto, la arquitectura de ladrillo vivió sus años de esplendor en la ciudad, en el periodo que abarca desde el último cuarto del siglo XIX hasta la tercera década del siglo XX, propiciado por una serie de circunstancias que concurrieron en la época:

- La fabricación masiva de ladrillos.

- La existencia de unos arquitectos dispuestos a utilizarlos en sus obras.

- Las referencias de la arquitectura latericia, en boga en las grandes capitales.

El primero de los factores es consecuencia de la existencia, en zonas cercanas a la ciudad, de cuantiosa materia prima apta para su elaboración, lo que propició el asentamiento de numerosas tejeras en la capital. Por lo que respecta a los arquitectos, fueron varios los que se afincaron en la ciudad, cada uno de ellos con unos rasgos propios, que dejaban patentes en los diseños de sus fachadas. Estos arquitectos, titulados en las Escuelas de Arquitectura de España, estaban al corriente de los nuevos materiales y de las posibilidades constructivas que permitían su uso, coincidiendo en el empleo de los ladrillos para ejecutar las fábricas y para elaborar los diseños ornamentales de las fachadas.

La consecuencia de estos hechos es la existencia de una abundante nómina de edificios de ladrillo al descubierto, la mayoría de ellos de uso residencial, que permite realizar un estudio técnico y de composición morfológica que desvele las características constructivas de esta época.

Todo el repertorio de inmuebles de ladrillo que fueron construidos en esta época forma parte del corpus ecléctico y modernista de la ciudad de Zamora, cuya notable entidad permi- tió la inclusión de la capital en la prestigiosa asociación Ruta Europea del Modernismo, en el año 2009.

\section{ANTECEDENTES}

La pertenencia de la ciudad de Zamora a dicha asociación internacional, acarrea la responsabilidad de divulgar y proteger este patrimonio. En este sentido, hay que reseñar que el estudio exhaustivo de los edificios de ladrillo, realizado por las autoras, permite sacar a la luz documentación muy precisa sobre las composiciones originales de las fachadas, sobre los diseños de la ornamentación y sobre los materiales con los que fueron proyectados y construidos. Esta información es fundamental en el ámbito de la rehabilitación y restauración de los inmuebles, ya que es un garante de que las intervenciones en los edificios, y de manera especial en los más emblemáticos, son acordes a la realidad constructiva originaria. No se debe obviar el hecho de que, hoy en día, un considerable número de inmuebles están desfigurados, fruto de las diversas actuaciones de las que han sido objeto a lo largo de los años.

La sensibilidad frente a la defensa del patrimonio histórico que ha mostrado el Consistorio, hecho que ha permitido recibir el Premio Nacional de la Real Fundación de Toledo del año 2012, junto con la pertenencia, como hemos apuntado, de la ciudad a la asociación Ruta Europea del Modernismo, son un revulsivo para estudiar constructiva y técnicamente las construcciones de esta época decimonónica, con el fin de conservar y recuperar el patrimonio ecléctico y modernista de la ciudad.

Asimismo, considerando que los inmuebles estudiados forman parte del Catálogo de Edificios Protegidos de la ciudad de Zamora, y que, a día de hoy, no existen estudios técnicos sobre ellos, la información procedente de los realizados a estos edificios de ladrillo puede servir de fuente documental que justifiquen las futuras intervenciones, como verdaderas evidencias de las composiciones de las fachadas y de los sistemas constructivos originales, de cara a una verdadera recuperación y mantenimiento de los edificios.

En concreto, se ha realizado el estudio a un total de 43 inmuebles, que son los que permanecen en la actualidad, de todos los que se construyeron con ladrillo al descubierto en la capital zamorana. Sus usos fueron diversos, de manera que el ladrillo fue empleado tanto en edificios fabriles, como en construcciones nobles, destacando entre éstos últimos el Instituto de Enseñanza Claudio Moyano y el Laboratorio Municipal. También fue empleado en los tres edificios más singulares de la ciudad, la Plaza de Toros, el Mercado de Abastos y el Cuartel de Caballería Viriato. No obstante el mayor porcentaje de construcciones latericias se concentró en residencias familiares y en casas de vecindad, situadas en el interior del casco amurallado.

\section{OBJETIVOS DEL ESTUDIO}

Dentro de este contexto y con el fin de que los datos que aporte la investigación proporcionen información para las futuras intervenciones para conservar el patrimonio existente se establecen los siguientes objetivos:

En primer lugar, conocer los diferentes tipos de ladrillos, su disposición y sistemas constructivos empleados en la construcción de los muros. 
Por otra parte establecer correlaciones mediante el análisis comparativo entre el uso de un determinado tipo de ladrillo, en un tipo concreto de edificaciones y con una composición constructiva determinada.

Por último y reconocido el vacío documental sobre las tipologías de las piezas latericias, de su morfología, de sus tamaños, de los aparejos empleados, de la disposición constructiva en los muros, e incluso, de la ejecución de las juntas, se pretende obtener un mejor conocimiento de los edificios y que puedan ser conocidos por los autores de los futuros proyectos de reforma en los mismos.

\section{LA FABRICACIÓN DEL LADRILLO: LAS TEJERAS}

Como se ha señalado anteriormente, una de las circunstancias que propició el desarrollo de esta arquitectura latericia fue la fabricación masiva de ladrillos, resultado de la llegada del desarrollo industrial que aprovechó la abundante materia prima, la arcilla, de los alrededores de la ciudad así como las tejeras que se fueron ubicando a extramuros del casco, agrupándose en determinados barrios de la capital.

Estos modestos talleres de cerámica fueron creciendo en número de una manera significativa, reconociéndose hasta un total de 10 (1) (2) (3) (4) (5) que desde 1855, año en el que se instauró el primero de ellos, y hasta 1910, pusieron de manifiesto las numerosas ventajas que para las construcciones de la zona ofrecía su implantación, tal y como lo expresaba en el primer expediente de solicitud de actividad su propietario José Carlos Escobar (6) que exponía que «el establecimiento de la fábrica ha de reportar al vecindario grandes ventajas ya por qué adquirirá a menor precio que los que hoy cuestan los objetos que en ella se construyan...».

Este aumento de las tejerías, tejeras y tejares, según la denominación mencionada en (7), discurría en paralelo al incremento de las construcciones con ladrillo en la ciudad, que comenzaba a tomar fuerza en este periodo, sustituyendo al tapial en las construcciones sencillas y a la piedra arenisca en los edificios más importantes.

Sin embargo, este crecimiento se frenó en la primera década del siglo XX, e incluso sufrió un importante declive, como lo demuestra la documentación de matrícula industrial de 1921 (8), que tiene catalogadas tan sólo cinco, de las diez que se habían relacionado veinte años atrás.

Tal descenso en la fabricación de cerámica era inversamente proporcional al crecimiento arquitectónico de la ciudad, ya que fue en este periodo de tiempo, entre 1900 y 1920 , cuando se produjo el mayor incremento de construcciones latericias.

La explicación a tal contradicción se encuentra en la ubicación a principios del siglo XX de la conocida como Tejera de San Antonio. Esta planta, de fabricación industrial, se asentó a los pies del yacimiento de Entrala, en El Perdigón, situada a tan sólo $15 \mathrm{~km}$, al sur de la capital y desplazó de la producción latericia a muchos de los talleres familiares que hasta ese momento surtían de ladrillos a los constructores de la ciudad. Esta fábrica tenía como principales ventajas su cercanía a la capital y una producción a mayor escala de ladrillos y tejas.

Las consecuencias de todo lo comentado se reflejan en las piezas empleadas en los distintos periodos. Tal es así que en las construcciones zamoranas de finales del S. XIX, se empleó el denominado ladrillo ordinario, incluso colocado al descubierto, que procedía exclusivamente de las tejeras artesanales. Por el contrario, a partir del año 1900, empezó a asomar en los edificios de la ciudad, el llamado ladrillo fino o prensado.

El carácter industrial de estos últimos, permitió fabricar, además de los anteriores, los ladrillos prensados o finos, de mejor factura, más regulares y de terminación más fina que los que se pudieran producir en los talleres artesanales. De hecho en la actualidad, la mayoría de los ladrillos que se ob-
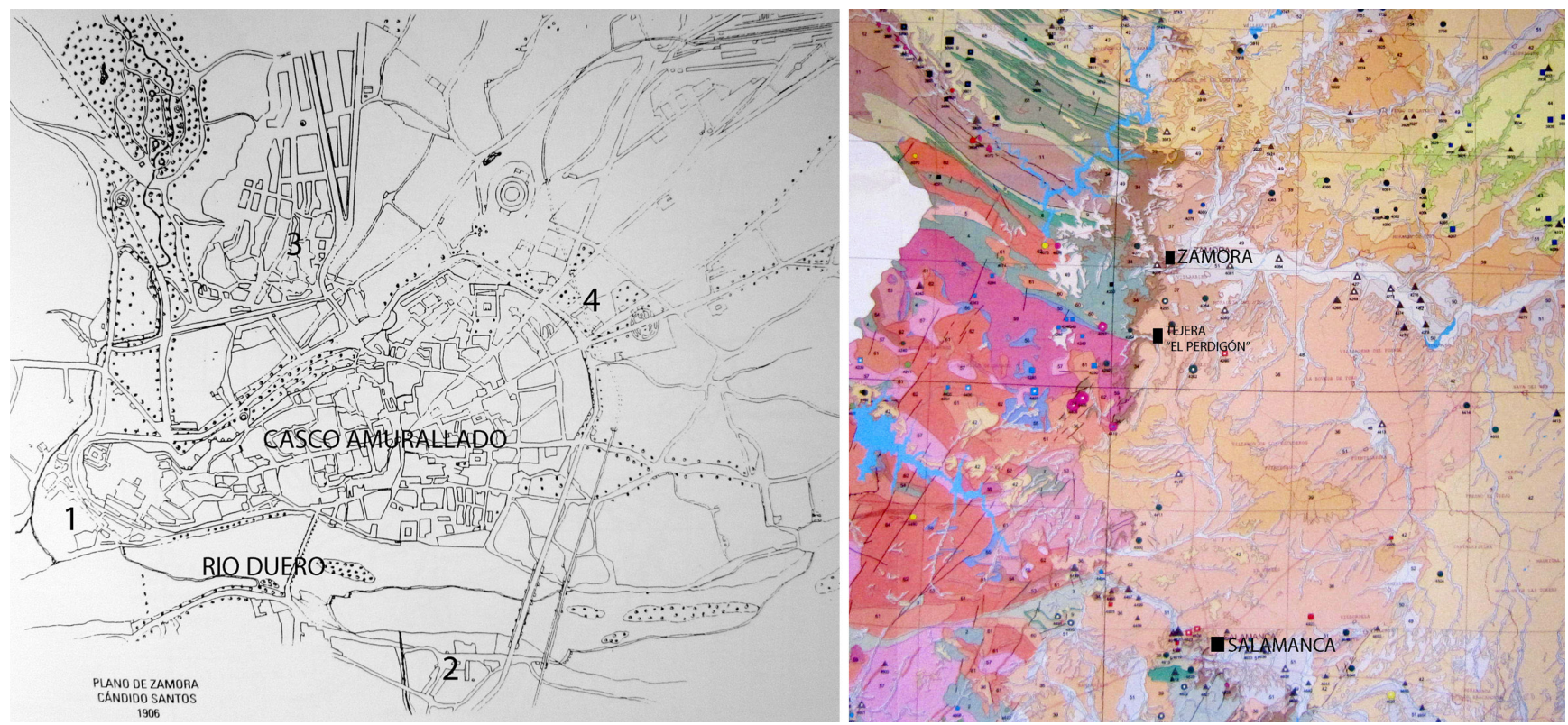

Figura 1. Izquierda. Plano de situación de las tejeras de la ciudad de Zamora (1855-1910). 1. Barrio de olivares (3 tejeras) - 2. Barrio de Pinilla (4 tejeras) - 3. Barrio de San Lázaro (2 tejeras) - 4. Ensanche (1 tejera).

Derecha. Plano de situación de la tejera de San Antonio, en el municipio de El Perdigón. 
servan en las construcciones de la capital zamorana son del tipo fino y proceden de la Tejera de San Antonio.

\section{LOS LADRILLOS}

Las primeras tejeras industriales fabricaban varios tipos de ladrillos mecánicos, el de extrusión, también llamado ordinario, (9) (10), que generalmente se revocaba, y el prensado, de mejor calidad, que se dejaba al descubierto y que también se denominaba de revestimiento o fino, como ya se ha comentado.

Esta clasificación de los ladrillos en ordinarios y finos o prensados proviene de su fabricación, de manera que los ladrillos finos fueron elaborados en prensas de estampa, de ahí su apelativo de prensados, mientras que los ordinarios eran aquellos que no eran prensados y que se fabricaban por extrusión.

No obstante, su fabricación no era el único de los motivos por los cuales el ladrillo era de un tipo u otro ya que el resultado tenía mucho que ver con la materia prima utilizada y con el celo con el que fueron fabricados (Figura 2).

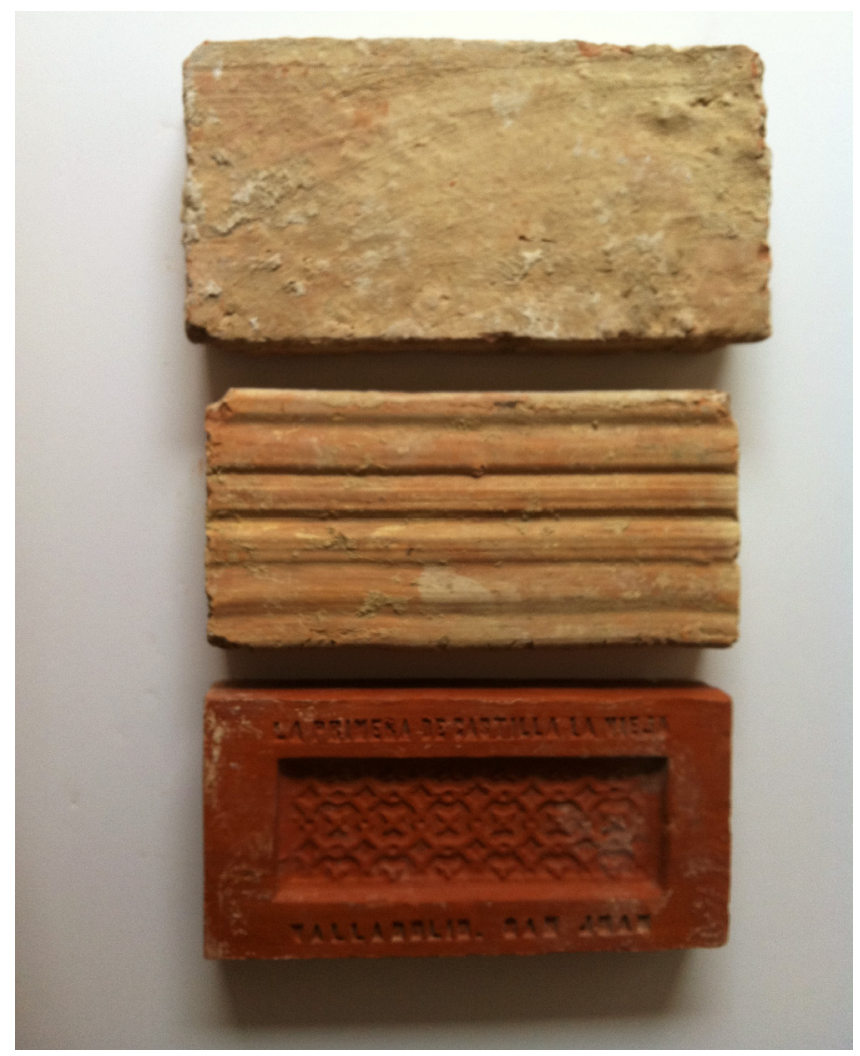

Figura 2. De arriba a abajo ladrillo tejar, ladrillo de galletera $\mathrm{y}$ ladrillo prensado de finales del siglo XIX principios del XX, procedentes de demoliciones.

\subsection{El ladrillo ordinario}

Según expone (11) el ladrillo ordinario era el que tenía una proporción mayor de arena en su composición, era más poroso y estaba fabricado con tierras poco arcillosas. Consecuencia de lo anterior y reconociendo que el aspecto externo del ladrillo ordinario no era el más idóneo para aplicarlo al descubierto en inmuebles de cierta entidad y tampoco poseía las características precisas de resistencia a la intemperie, en la mayoría de las obras éste fue revocado. Tan sólo fue deja- do al descubierto en las fachadas de edificios industriales, en construcciones poco nobles y en residencias levantadas con anterioridad a 1900.

Por su parte, la fabricación industrial o mecánica de los ladrillos ordinarios, tal y como reconoce (10), difiere del proceso artesanal, dando lugar a un "Ladrillo ordinario. - De mejores pastas y cocción mas igual que el fabricado en hormigueros, mejor cortado y más resistente».

A su vez este mismo proceso industrial permitió que se fabricaran ladrillos con diseños innovadores cuya finalidad principal perseguía mejorar la adherencia con el mortero. De esta manera, comenzaron a producirse ladrillos con las tablas acanaladas o estriadas, resultando una imagen diferente en las testas dejadas al descubierto, respecto al tratamiento del grueso de junta y la calidad del ladrillo que quedaba a la vista, sin revocar (Figura 3 superior).

No obstante la característica más destacada de estos ladrillos es la heterogeneidad cromática, por lo que era habitual que los entrepaños de las fachadas estuvieran compuestos por piezas de diversos colores. Tal es así que entre los ladrillos de varias gamas de rojos y anaranjados que muestran las fábricas, se intercalaban otros de tonos negruzcos, conocidos como ladrillos santos (11), cuyo color no provenía de las arcillas con las que habían sido fabricados, sino que era el resultado de su cocción, ya que habían sufrido un exceso de cochura (Figura 3 inferior).
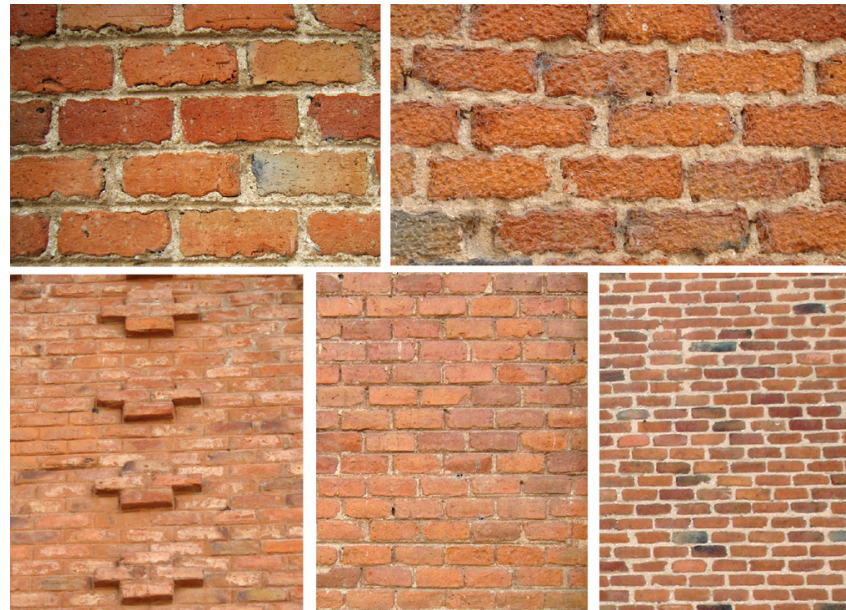

Figura 3. Distintos ejemplos de la ejecución de la fábrica vista, respecto al tratamiento del grueso de junta y la calidad del ladrillo que queda a la vista, sin revocar.

Superior: fábrica de ladrillo ordinario con las tablas acanaladas. Izquierda: cuartel de Caballería Viriato, Ingeniero Francisco Vida

(1919). Derecha: fachada lateral de la Casa de Gabino Bobo del arquitecto Segundo Viloria (1916). Inferior: ladrillos ordinarios, que muestran una clara heterogeneidad cromática, y diferente ejecución

de las juntas. Izquierda: casa de Bernardo Ballesteros (1889).

Central: mercado Municipal de Abastos (1902). Derecha:

fábrica de Harinas «Gabino Bobo» (1907). Todas las obras del arquitecto Segundo Viloria.

Centrándonos en la categoría de acabados, en el conjunto de los inmuebles construidos con ladrillos ordinarios se muestran claramente dos clases de piezas, que revelan sus diferencias tanto en el perfilado de sus ángulos como en la planeidad de sus caras. En este sentido, y a diferencia de lo esperado, la utilización de ladrillos ordinarios de mejor o peor calidad no tenía relación con la cronología de la fabricación sino que 
dependía del uso del inmueble, de manera que los edificios destinados a industrias y talleres fueron construidos con ladrillo de peor factura que los de uso residencial.

A la calidad del ladrillo ordinario se unen otras dos características de las fábricas que evidencian que las fachadas de ladrillo de las residencias fueron construidas con mayor esmero que las de las industrias. Una de estas características tiene que ver con la ejecución del aparejo, de manera que, en el primero de los usos, el residencial, se observa una correcta alineación de las llagas y los tendeles de las juntas del mortero, mientras que en los edificios industriales, se observan desviaciones, sobre todo en las llagas. La otra característica tiene que ver, precisamente con el tipo de junta realizada, puesto que, en las viviendas el mortero apenas se aprecia al exterior y está perfilado, mientras que en los edificios de uso industrial la junta tiene un espesor superior a los $5 \mathrm{~mm}$ y en muchos casos, cercano a los $10 \mathrm{~mm}$ (Figura 4).

De igual forma y realizando un análisis específico del uso del ladrillo ordinario en la ciudad de Zamora se ha observado que al descubierto se empleó mayoritariamente en la ejecución de edificios industriales y talleres, y sólo se utilizó en inmuebles residenciales en las décadas del siglo XIX, ante la ausencia de fabricación de ladrillos finos en aquella centuria, tanto en la ciudad como en sus alrededores.

\subsection{El ladrillo prensado o fino}

Tal y como expone (10) el ladrillo fino o prensado se reconocía fabricado con arcillas escogidas y arenas muy finas, estando bien cocidos y obteniéndose piezas de gran igualdad. Debido al proceso de prensado las superficies eran muy compactas y resistentes a la acción de los agentes atmosféricos, lo que los predisponía para ser empleados preferentemente en los paramentos exteriores en fachada al descubierto.

Desde el punto de vista morfológico eran reconocidos por estar perfectamente moldeados y perfilados, siendo de aristas vivas y superficies tersas, de manera que para favorecer la adherencia con el mortero se dejaban en sus planos, huecos o cavidades que incorporaban dibujos, estrías, cuadrículas o letreros.

A diferencia de los descritos en el apartado anterior, estos ladrillos, elaborados industrialmente, se caracterizaban por tanto por ser más homogéneos en sus medidas, por tener un colorido similar y por tener las «dos caras de asiento rebajadas, ..., marcando el nombre comercial» (12). El «singular» diseño permitía de esta forma identificar la tejera de procedencia, pero además y desde un punto de vista estrictamente constructivo recogía el mortero para disminuir el espesor de las juntas y tal y como se ha comentado facilitaba la adherencia con este material auxiliar. La Tejera de San Antonio fue la única que adoptó esta práctica y estampaba su nombre y el lugar de procedencia en las dos tablas de los ladrillos que fabricaba (Figura 5 superior). Complementariamente esta morfología del ladrillo fue, en ocasiones, utilizada como recurso ornamental de manera que los arquitectos lo colocaban en las impostas, en las cornisas y en las jambas, dejando vistas las estampas labradas.

El rebaje en las tablas de los ladrillos daba como resultado un aparejo con una apariencia completamente diferente a la que procedía de la traba con el ladrillo ordinario, ya que, mientras que con éste las juntas eran gruesas y el mortero asomaba al exterior, con el ladrillo fino las juntas quedaban ocultas, ya que el mortero no salía hasta el exterior, y con un espesor que no superaba los $0,5 \mathrm{~cm}$.

No obstante, a pesar de que estos ladrillos finos propiciaban la ejecución del muro con juntas ocultas, también se levantaron fábricas con juntas enrasadas, en las que, a pesar de tener poco espesor, el mortero asomaba al exterior. Muestra de lo comentado se reconoce en la documentación incluida en diversos proyectos originales como es el caso del Laboratorio Municipal (1909), elaborado por el arquitecto Francisco Ferriol (13), en el que a pesar de utilizar un ladrillo «de clase superior procedente de la Tejera de El Perdigón, será ladrillo prensado» según cita en el Pliego de Condiciones, las juntas fueron enrasadas. (Figura 5 inferior).

A partir de 1900, los ladrillos empleados al descubierto en los inmuebles residenciales fueron, en todos los casos, ladrillos finos o prensados, incluso en las viviendas más modestas, a pesar de que eran más costosos que los ordinarios. No cabe duda que este hecho está relacionado con la imagen de belleza al exterior y con las características físicas y mecánicas que presentan frente a los agentes atmosféricos, al estar expuestos al exterior.

\subsection{Los ladrillos aplantillados y otras piezas ornamentales}

Complementariamente al uso del ladrillo fino de morfología convencional, y coincidiendo en la misma época, se fabricaron otro tipo de piezas especiales que recibían el nombre de ladrillos aplantillados. Según establece (12) éstas constituían
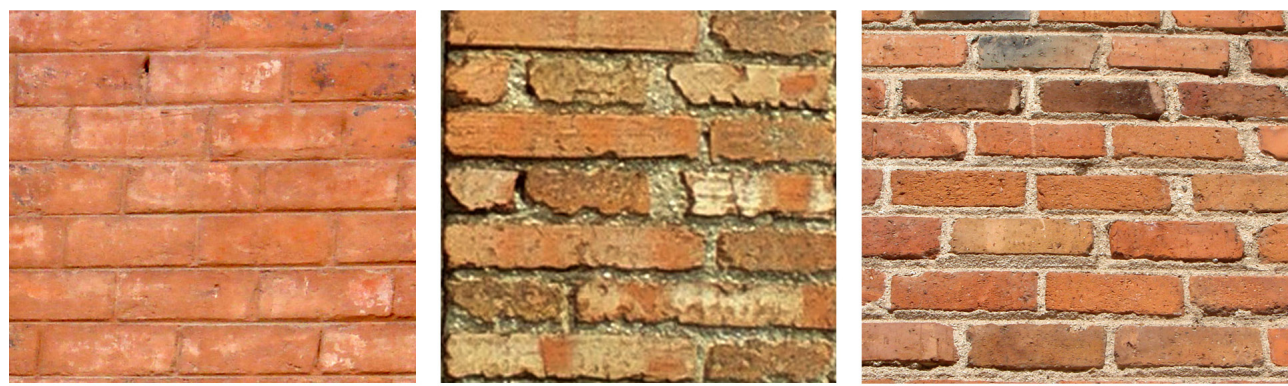

Figura 4. Aparejos de ladrillos ordinarios, con la traba más cuidada y las juntas más estrechas en edificios residenciales que en los industriales. Izquierda: aparejo a tizón, Casa de Matilde Merchán, del arquitecto Segundo Viloria (1894). Central: aparejo belga, Fábrica de harinas Isidoro Rubio, del arquitecto Gregorio Pérez Arribas (1917). Derecha: aparejo a tizón, Matadero Municipal, de los arquitectos Enrique Crespo y Francisco Hernanz (1929). 

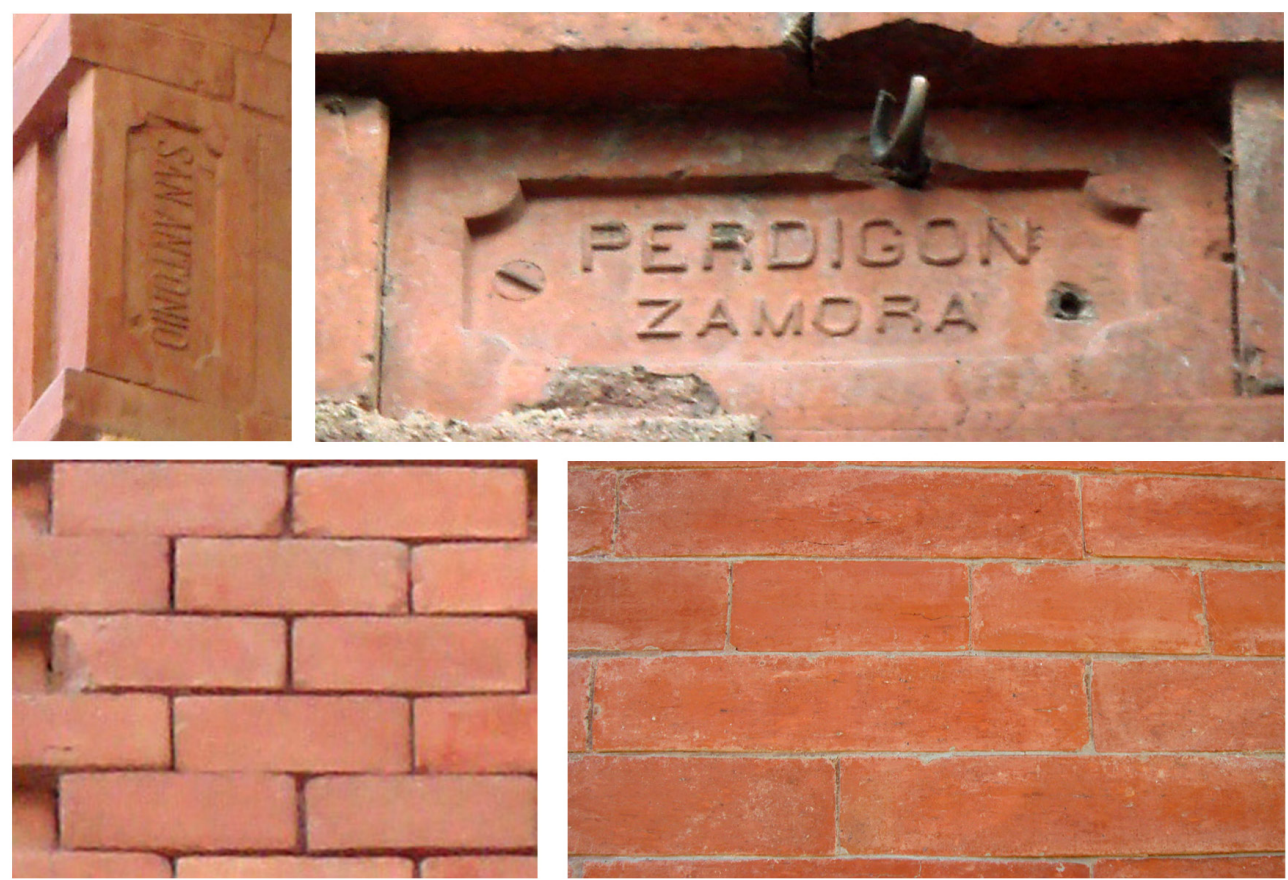

Figura 5. Superior: ladrillos fabricados en la Tejera de San Antonio, en El Perdigón (Zamora). Izquierda: jamba de un vano de un edificio del arquitecto Francisco Ferriol (hacia 1910). Derecha: En imposta de un edificio del arquitecto Segundo Viloria (hacia 1910).

Inferior izquierda: aparejo con ladrillo fino con junta a hueso u oculta, Casa de José Cid del arquitecto Segundo Viloria (1902). Inferior derecha: aparejo con ladrillo fino y junta enrasada, Laboratorio Municipal del arquitecto Francisco Ferriol (1909).

una variedad que tenían la singularidad de poseer una geometría diferente a la paralelepipédica.

Eran fabricados con prensa, ya que constituían una variedad de los llamados finos o prensados, por lo que coinciden con todo lo especificado para ellos desde el punto de vista composicional y de fabricación.

Estos ladrillos aplantillados, denominados así por los propios técnicos, se fabricaban con moldes y se elaboraban por encargo, conforme a las necesidades y los diseños específicos de cada obra. Una prueba de esta afirmación está en las aclaraciones que sobre estas piezas escribió el arquitecto Francisco Ferriol (13) en el Pliego de Condiciones del Proyecto del Laboratorio Municipal (1909), en el cual, refiriéndose a los ladrillos, expone que «...para los arcos y molduras de los antepechos deberá ser aplantillado para seguir la forma del arco. El contratista mandará hacer los moldes ajustados á las plantillas para estos arcos y molduras».

En concreto para el ejemplo citado, los ladrillos aplantillados tenían forma troncocónica porque se utilizaron en la ejecución de los sardineles de los arcos, con el fin de resolver las diferencias métricas entre el intradós y el extradós. Este recurso constructivo también fue utilizado por otros arquitectos afincados en Zamora, como Segundo Viloria en cuyos trabajos se puede observar la existencia de piezas acuñadas en los salmeres y en las contraclaves de los arcos, que absorben los ángulos agudos de los apoyos tal y como se muestra en la (Figura 6 superior).

Adicionalmente se colocaban piezas especiales ornamentadas, generalmente con motivos vegetales que ocupaban las claves de los arcos, siendo habitual encontrarlas en los vanos adintelados de las obras del arquitecto Segundo Viloria.
Otra aplicación característica de las piezas aplantilladas fue en la ejecución de las cornisas, cuyas formas en ménsula permitían conseguir un mayor vuelo frente a los de una sola hilada. Este sistema fue utilizado por el arquitecto Gregorio Pérez Arribas, siendo una técnica poco extendida entre el resto de los arquitectos afincados en la ciudad (Figura 6 inferior).

Los ladrillos aplantillados y las piezas ornamentales de las construcciones zamoranas fueron de diseños y aplicaciones más comedidos que los reconocidos en otras ciudades en las que las representaciones de los relieves tenían formas humanas y las cornisas eran más pronunciadas y esbeltas. La Tejería Mecánica de D. Eloy Silió, de Valladolid, fabricaba ladrillos aplantillados de dimensiones superiores a las normales para aleros y cornisas.

\subsection{Tipos y tamaños de los ladrillos}

Con el fin de establecer una relación entre los usos de los inmuebles, los tipos de ladrillos que se colocaron al descubierto en los inmuebles zamoranos en la época de estudio y los tamaños de sus caras, se ha elaborado una tabla con una clasificación cronológica, en la que se han incluido todos los inmuebles con los datos citados (Tabla 1).

El análisis de dicha tabla deja patentes varios aspectos que fundamentan las afirmaciones dadas con anterioridad. En este sentido, el hecho de que tan sólo los inmuebles residenciales construidos con anterioridad a 1900 fueran levantados con ladrillo ordinario al descubierto, ratifica la inexistencia de ladrillos finos hasta ese momento. Esto se afirma tras observar que a partir de este momento, todos los demás inmuebles con ese uso fueron construidos con ladrillo prensado. Además, este material más fino y regular también se utilizó en otros edificios de uso selecto como fueron el Instituto de 

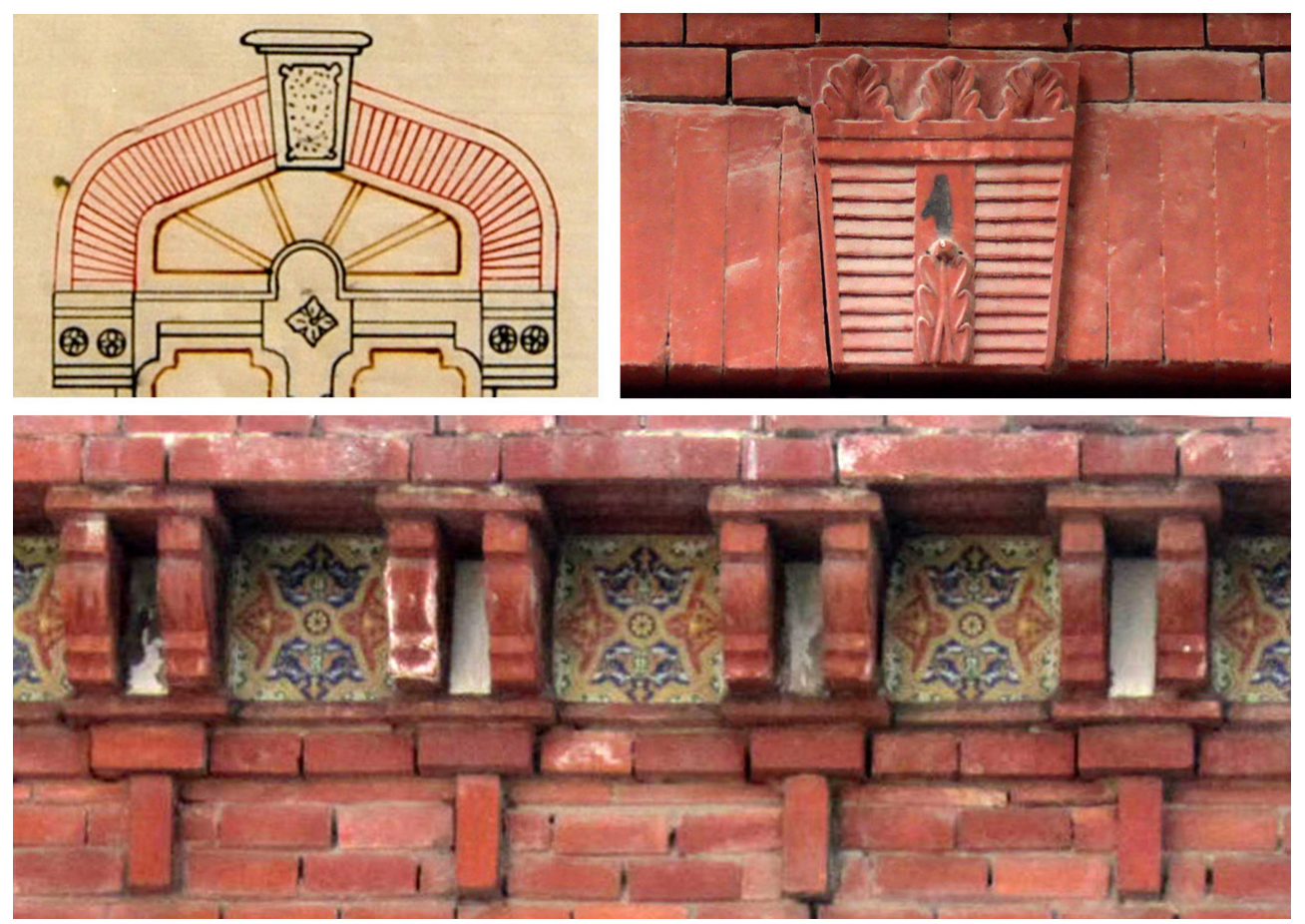

Figura 6. Superior izquierda: detalle del arco del Proyecto original del Laboratorio Municipal, del arquitecto Francisco Ferriol (1909). Superior derecha: fotografía del estado actual detalle de la clave y de las contraclaves de la casa de Augusto Millán, del arquitecto Segundo Viloria (1914). Inferior: alero de ladrillos aplantillados. Casa de Miguel Hernández, del arquitecto Gregorio Pérez Arribas (1908).

Educación Secundario Claudio Moyano (1902) o el Colegio Arias Gonzalo (1929).

Por otra parte, los edificios industriales, los talleres, los almacenes y otros de usos similares, como fueron el Mercado de Abastos (1902), el Cuartel de Caballería Viriato (1919), el Lavadero Municipal (1928) y el Matadero Municipal (1929) se construyeron con ladrillos ordinarios al descubierto. Esto se justifica, en primer lugar por una cuestión económica, pues el ladrillo ordinario era más barato que el prensado y en segundo lugar a una cuestión de acabado, ya que su desigual terminación y su textura más tosca les conferían un aspecto más sobrio y por lo tanto más apropiado para este tipo de inmuebles.

Por lo que respecta al tamaño de los ladrillos, se observa una amplia diversidad de medidas de las tres caras de las piezas, tanto en los ordinarios como en los finos, siendo aquellos los que acusan mayores diferencias. No obstante, a pesar de que las variaciones en los ladrillos prensados no exceden de los 2 $\mathrm{cm}$, esta diferencia es suficiente para poder afirmar que hasta el final del periodo que abarca este estudio (1929), en la capital zamorana no se utilizaron ladrillos estandarizados, ni tan siquiera aquellos que habían sido fabricados industrialmente.

Por otra parte y por la facilidad visual que ofrece la fábrica al descubierto, en el presente artículo y una vez conocidos los distintos tipos de ladrillo (ordinario y fino) y su utilización en diferentes edificios y usos, se ha realizado un análisis exhaustivo sobre el dimensionado de las piezas, con el fin de establecer una posible correlación entre el uso de los inmuebles, el tipo de ladrillo y el dimensionado de las mismas.

En relación al dimensionado, estudios previos realizados en otras ciudades limítrofes a Zamora, como es el caso concreto de Valladolid (14), reconocen la fabricación de ladrillos con unos tipos de formato determinado de la Tejería Mecánica de D. Eloy Silió. Éstos, en función de sus medidas, eran designados con los apelativos de Borgoña (22,0 × 10,7 a 11,0 $\times$ $5,4$ a $5,5 \mathrm{~cm})$, Castilla $(26,5 \times 13,0 \times 5,8 \mathrm{~cm})$ y España $(25,5$ $\times 12,5 \times 4,8 \mathrm{~cm})$.

Mayoritariamente el empleado en las construcciones de la capital fue de dimensiones $25,0 \times 12,0$ a $12,5 \times 4,0$ a 5 , o que no corresponde a ninguno de los tipos apuntados anteriormente. No obstante, sí fue tipificado como ladrillo prensado en una clasificación realizada por Foerster (15), en su manual, lo que pone de manifiesto que estas medidas fueron habituales en las construcciones del país.

Se observa que los utilizados en las obras zamoranas se podrían ajustar a los denominados España, no habiéndose reconocido ni un solo ejemplo, en el presente estudio, de los designados como Castilla y encontrando un solo edificio en la capital del tipo Borgoña, en la casa de Eduardo Montero (1910) del arquitecto Francisco Ferriol.

Cabe destacar que no se han encontrado muestras de edificios construidos con ladrillos finos más largos, cuyas sogas (longitud de la pieza) tuvieran dimensiones de $26 \mathrm{~cm}$. Sin embargo, estas piezas sí se fabricaban y se empleaban en construcciones de otras ciudades como fue el caso de Segovia, cuyas dimensiones alcanzaban hasta $30 \mathrm{~cm} \mathrm{(16).}$

\section{EJECUCIÓN DEL MURO}

Por último en el análisis realizado sobre la fábrica de ladrillo en los edificios de Zamora se han estudiado los aspectos relativos al tipo de aparejo y al sistema de ejecución utilizado en la construcción de sus muros. 
Tabla 1. Los tamaños de los ladrillos se nombran: soga $\times$ tizón $\times$ grueso.

\begin{tabular}{|c|c|c|c|c|c|}
\hline \multirow{2}{*}{ EDIFICIO } & \multirow{2}{*}{ AÑO } & \multicolumn{3}{|c|}{ FORMATO DE LOS LADRILLOS } & \multirow{2}{*}{$\begin{array}{c}\text { TIPO DE } \\
\text { LADRILLO }\end{array}$} \\
\hline & & soga & tizón & grueso & \\
\hline \multirow{2}{*}{ Plaza de Toros } & \multirow{2}{*}{1884} & 21 & 12,5 & 3,5 & \multirow{2}{*}{ Ordinario } \\
\hline & & $26-27$ & 13,5 & 4,2 & \\
\hline Casa Ballesteros & 1889 & 25 & 14 & 6 & Ordinario \\
\hline Casa Merchán & 1894 & 26 & 14 & 6 & Ordinario \\
\hline Casa A. Bobo & 1898 & $21-23$ & $10-13$ & $4,2-5,0$ & Ordinario \\
\hline Casa Gallego & 1900 & 25 & 12,5 & $4,5-5$ & Prensado \\
\hline $\begin{array}{l}\text { Central eléctrica } \\
\text { El Porvenir de Zamora }\end{array}$ & 1901 & 24 & 12 & 5 & Prensado \\
\hline Instituto Claudio Moyano & 1902 & 25 & 12 & 4,8 & Prensado \\
\hline Casa Cid & 1902 & 24,5 & 12 & $4-5$ & Prensado \\
\hline \multirow{2}{*}{ Mercado de Abastos } & \multirow{2}{*}{1902} & 21 & 13 & 5 & \multirow{2}{*}{ Ordinario } \\
\hline & & 27 a 28 & 14 & $5-6$ & \\
\hline Fábrica de harinas Bobo & 1907 & $26-27$ & $13-13,5$ & $4,5-5$ & Ordinario \\
\hline Casa Salvador & 1908 & 25 & $12-12,5$ & $5-5,2$ & Prensado \\
\hline Casa de G. Prada & 1908 & 25 & 12 & 4 a 4,2 & Prensado \\
\hline Casa de M. Horna & 1908 & 26 & 12 & $4,5-6$ & Prensado \\
\hline Casa J. N. & 1907 & 25 & $12-12,5$ & $4,8-5,0$ & Prensado \\
\hline Casa Rábano & 1909 & 25,5 & $12-12,3$ & 4,3 a 4,6 & Prensado \\
\hline Laboratorio Municipal & 1909 & 24,5 & 12 & 4,7 & Prensado \\
\hline Almacén Sdad. García Hnos & 1910 & $22-22,5$ & 11 & 5 & Ordinario \\
\hline Casa Montero & 1910 & 22 & 11 & 5 & Prensado \\
\hline Cercado de M. Hervella & 1911 & 25,5 & 12,5 & 4,5 & Prensado \\
\hline Casa Millán & 1914 & 26 & 12,5 & 4,5 a 5 & Prensado \\
\hline Casa Taller Laguna & 1913 & 28 y 27 & 13 & 5 & Ordinario \\
\hline Casa G. Bobo & 1916 & 25 & 12 a 15 & $4-4,7$ & Prensado \\
\hline Fábrica de harinas Rubio & 1916 & 26 & $12-14$ & $5-5,5$ & Ordinario \\
\hline Cuartel de Caballería Viriato & 1919 & $21,5-22,5$ & $13-13,5$ & 4,8 & Ordinario \\
\hline Panteón Alonso & 1921 & 26 & 12,5 y 13 & $4,5^{-a} 4,7$ & Prensado \\
\hline \multirow{3}{*}{$\begin{array}{l}\text { Fábrica de harinas } \\
\text { La Panera Social }\end{array}$} & \multirow{3}{*}{1921} & 26 & 16 & 6 a 6,5 & \multirow{3}{*}{ Ordinario } \\
\hline & & 26 & $14-18-19,5$ & & \\
\hline & & 25 & $11,5-12,5$ & $4-5-8$ & \\
\hline Casa Amigo & 1922 & 24 & $12-14$ & $4,5-5,5$ & Prensado \\
\hline Casa Lozano & 1923 & 25 & 12 & 4,5 & Prensado \\
\hline Colegio Arias Gonzalo & 1929 & 22,5 a 26 & 12,5 a 13 & $4,0-4,7$ & Prensado \\
\hline Lavadero Municipal & 1928 & $22-23$ & $11-11,5$ & $4-4,3$ & Ordinario \\
\hline Matadero Municipal & 1929 & $26-27$ & $12-13,5$ & 5 & Ordinario \\
\hline
\end{tabular}

Es evidente que las características físicas de los ladrillos y su adecuada utilización en los paramentos eran sobradamente conocidas por los arquitectos que trabajaron en la capital zamorana en aquella época, hecho que se manifiesta en las numerosas construcciones que permanecen en pie en la actualidad y que están perfectamente documentadas en las memorias de los proyectos redactados.

$\mathrm{Al}$ igual que en los apartados anteriores, son significativas las diferencias existentes antes y después del año 1900, de tal forma que según describe (17) aquellos proyectos que fueron redactados a finales de siglo describen la construcción indicando que se empleará "fábrica de ladrillo al descubierto", sin especificar el tipo de ladrillo a utilizar, ordinario o prensado. Sin embargo, en las obras posteriores, que fueron proyectadas a partir de 1900, el mismo arquitecto que anteriormente obviaba determinar el tipo de ladrillo, destacaba de forma significativa el empleo de los dos que han sido apuntados con anterioridad, describiendo que las fachadas se constituirían con «fábrica de ladrillo fino trasdosado con ladrillo ordinario» (18).
Esta sencilla descripción que aparece en las memorias de los proyectos detalla de manera concisa el sistema de ejecución de los muros de las fachadas que se utilizaba en la época de estudio. Este sistema consistía en construir muros careados, fuertemente trabados entre sí, en los que la cara exterior la componía una hoja de ladrillo fino, que se trasdosaba por el interior (19) con fábrica de ladrillo ordinario hasta completar la anchura del muro deseada. Lo que pretendía este sistema constructivo era levantar una sola hoja, construida con dos materiales diferentes, mediante una perfecta trabazón entre ambas, siguiendo el sistema habitual de construir muros de espesores importantes tal y como describe Villanueva (20).

Para conseguir este sistema, los ladrillos que marcaban las dimensiones eran los ladrillos finos, colocados al exterior, a cuyo grosor se acomodaban los ordinarios, colocados en el interior, motivo por el cual, éstos últimos deberían ser de menor canto para poder ser introducidos entre los resquicios que dejaban los ladrillos prensados. De esta manera, las juntas del aparejo exterior eran finas y las del interior eran más gruesas. 
La cara exterior de estos muros de cerramiento se ejecutaba con esmero, siguiendo la teoría de la corriente racionalista (21) que se desplegaba por todo el territorio español, de manera que el ladrillo también fue aceptado por los arquitectos afincados en Zamora como un material digno de ser tomado en consideración, para derivar de él todo un sistema arquitectónico, aplicándolo como parte integrante del aparato ornamental de las fachadas.

Con tal fin, la construcción de la hoja exterior pasaba por una cuidada y estudiada disposición de los ladrillos, combinando piezas enrasadas, con otras resaltadas y rehundidas, y a su vez, asentando las piezas en todas las posiciones posibles, a soga, a tizón, a sardinel, a serreta, etc., dando lugar a una gran variedad de combinaciones geométricas. Esta situación generaba un desorden en la cara interna, que tenía que ser absorbido por la hoja interior. Por lo tanto, ésta quedaba tan sólo como el complemento matérico y estructural del cerramiento, cuya ejecución consistía en rellenar los huecos que iba dejando la hoja exterior y así conseguir una perfecta trabazón entre ambos, sin más orden que el que le imponía la construcción de la cara externa. Para conseguirlo, se introducían piezas recortadas que complementaran los vacíos, sin ejecutar un aparejo concreto (Figura 7 superior).
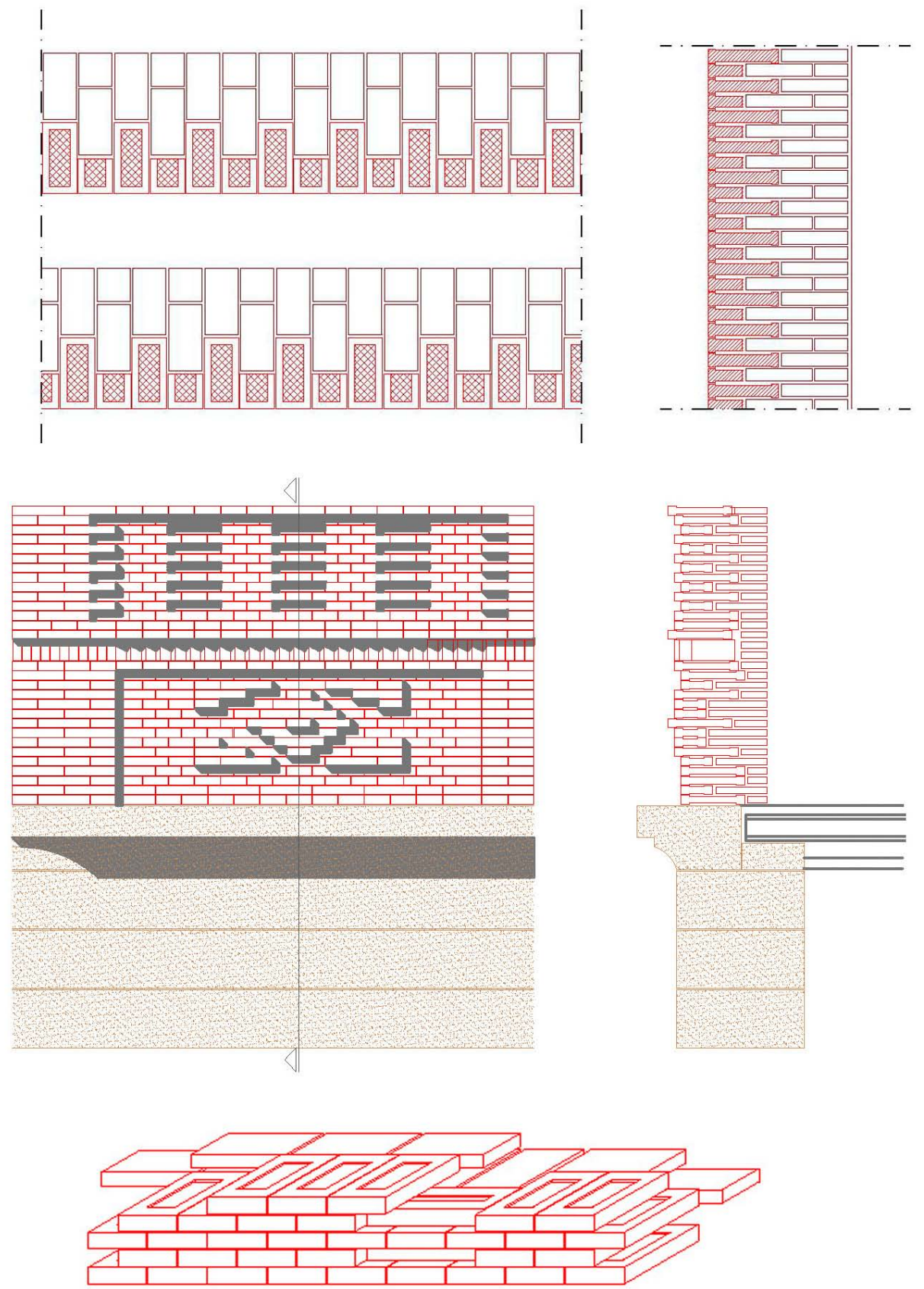

Figura 7. Superior: secciones horizontal y vertical del muro careado trabado, el exterior de ladrillo fino, a tizón. La interior de ladrillo ordinario, completando los vacíos hasta conseguir la anchura total. Izquierda: planta de dos hiladas consecutivas, hilada par e hilada impar. Derecha: sección vertical. Central e inferior: alzado/sección y detalle de trabazón de las dos hojas de ladrillo, la exterior de ladrillo fino con piezas resaltadas, rehundidas, a soga, a tizón y a sardinel y la interior de ladrillo ordinario. Central: alzado de un entrepaño y sección vertical del muro, mostrando las hiladas alternas. Inferior: sección horizontal con la formación del aparejo (Dibujos de autoría propia). 
Cuando la cara exterior contenía entrepaños carentes de ornamentación, la reorganización de la interior consistía en ir alternando las piezas de manera inversa, para complementarse, empleando también en la cara oculta un aparejo ordenado (Figura 7 central e inferior).

\section{CONCLUSIONES}

El ladrillo comenzó a usarse en las construcciones zamoranas a partir del último cuarto del siglo XIX, y se instauró como elemento constructivo y ornamental por excelencia. Este hecho coincidió con el resurgir de la ciudad y con la implantación de numerosas tejeras en los barrios de la ciudad, derivado de la existencia de abundante materia prima en zonas cercanas a la capital. En estos talleres se elaboraban tan sólo ladrillos ordinarios hasta que, en el cambio de siglo, se asentó una tejera industrial, que trajo un aumento de la producción y la fabricación del ladrillo fino o prensado.

En la construcción de las fachadas se emplearon los dos tipos de ladrillo nombrados, por un lado el llamado fino o prensado, elaborado en prensa, con arcillas escogidas, que tenía un acabado de factura más cuidada y mejor comportamiento frente a los agentes atmosféricos, por lo que era el idóneo para dejarlo al descubierto. Sin embargo, en los edificios industriales y almacenes se utilizó de manera exclusiva, el ladrillo ordinario, más tosco pero también más económico que le aportaba una imagen más apropiada a este tipo de usos.

También se fabricaron ladrillos de formas diferentes al paralelepípedo denominados ladrillos aplantillados que resolvían determinados elementos constructivos, como cornisas, arcos o pilastras en los que la cara moldurada se dejaba vista como motivo ornamental. Eran ladrillos prensados.

La multiplicidad de tejeras derivó en una gran variedad de tamaños y tonos de los ladrillos, heterogeneidad que no se terminó con la mecanización del producto. No obstante, la fabricación industrial elaboró ladrillos finos con las tablas rebajadas, para recoger el mortero del aparejo y conseguir ejecutarlo con juntas ocultas, hasta ese momento, inexistentes en la arquitectura zamorana.

Respecto a los tamaños de los ladrillos, no había un formato común, si bien, en general, las medidas se acercaban a las piezas fabricadas en ciudades cercanas, con medidas entre 24 y $26 \mathrm{~cm}$ de soga.

Por lo que se refiere a la ejecución, los arquitectos afincados en la ciudad eran conocedores de las técnicas constructivas de los ladrillos y de la corriente racionalista, construían el muro careado mediante dos caras fuertemente trabadas, la exterior de ladrillo fino al descubierto, de cuidada composición, que se trasdosaba a la interior con el ladrillo ordinario.

\section{REFERENCIAS}

(1) Pérez, E. J. (1895). Guía del viajero en Zamora. p. 16. Zamora: Imprenta Provincial.

(2) Gago-Vaquero, J. L. (1988). La arquitectura y los arquitectos del ensanche Zamora 1925-1950. p. 122. Zamora: Instituto de Estudios Zamoranos Florián de Ocampo.

(3) Archivo Histórico Provincial de Zamora. (1896). Solicitud de licencia de actividad para una tejera. Obras, sign. O.XIX-19/IX.

(4) Gago-Vaquero, J. L. (1988). La arquitectura y los arquitectos del ensanche Zamora 1925-1950. p. 110. Zamora: Instituto de Estudios Zamoranos Florián de Ocampo.

(5) Gago-Vaquero, J. L. (1988). Plano de la España Regional de 1910. En La arquitectura y los arquitectos del ensanche Zamora 1925-1950, (documentación adjunta). Zamora: Instituto de Estudios Zamoranos Florián de Ocampo.

(6) Archivo Histórico Provincial de Zamora. (1855). Escrito de solicitud de licencia de obras. Obras, sign. 664-04.

(7) Paniagua, J.R. (1987). Vocabulario básico de arquitectura. Madrid: Cátedra.

(8) Archivo Histórico Provincial de Zamora. (1921-1922). Libro de matriculas de industrial.

(9) Matallana, M. (1848). Vocabulario de Arquitectura Civil. p. 282, Madrid: Imprenta a cargo de Don Francisco Rodríguez.

(10) Rebolledo, J.A. (1910). El manual del constructor. ( $5^{\mathrm{a}}$ ed. corregida, reformada y aumentada por Ribera, E.). Madrid: Sáenz de Jubera Hermanos.

(11) Antuña, J. (2009). Léxico de la construcción. p.284. (Reedición). Madrid: Instituto Eduardo Torroja de la Construcción y el Cemento.

(12) Reverté, P. (1916). La industria ladrillera. Moldeo manual y mecánico de los ladrillos, secaderos y hornos modernos. Normas para el empleo de los ladrillos en las obras. Barcelona: Gustavo Gili. S.A.

(13) Archivo Histórico Provincial de Zamora. (1909). Proyecto original del laboratorio municipal del arquitecto Fracisco Ferriol. Obras, sign. O.20-1/VII.

(14) Camino, M. S. (2001). Construcción y ornamentación de las fachadas de ladrillo prensado, al descubierto, en la ciudad de Valladolid (Tesis doctoral). Valladolid: ETSA - Universidad de Valladolid. http://www.cervantesvirtual.com/nd/ ark:/59851/bmcr78b9.

(15) Foerster, M. (1926). Manual de Ingeniero Constructor y del Arquitecto. (40 a edición). Barcelona: Espasa Calpe.

(16) Barré, L.A. (1901). Pequeña enciclopedia práctica de construcción, Tomo $n^{o} 2$ Materiales de Construcción. Madrid: Editorial Mailly_Baillière e Hijos.

(17) Archivo Histórico Provincial de Zamora. (1894). Proyecto original de la casa de Matilde Merchán del arquitecto Segundo Viloria. Obras, sign. 692-4.

(18) Archivo Histórico Provincial de Zamora. (1902). Proyecto de la casa de José Cid del arquitecto Segundo Viloria. Obras, sign. 713-12. 
(19) Antuña, J. (2009). Léxico de la construcción. (Reedición). Madrid: Instituto Eduardo Torroja de la Construcción y el Cemento.

(20) Villanueva, J. (1827). Arte de Albañilería o instrucciones para jóvenes que se dediquen á él, en que se trata de herramientas necesarias del albañil, formación de andamio, y toda clase de construcciones. Madrid: Edición en la oficina de Don Francisco Martínez Dávila.

(21) Adell-Argilés, J. M. (1986). Arquitectura de ladrillos del siglo XIX. Técnica y Forma. p. X. Madrid: Fundación Universidad-Empresas. 